\title{
Atenção ao parto por enfermeira obstétrica em maternidades vinculadas à Rede Cegonha, Brasil - 2017
}

\author{
Delivery care by obstetric nurses in maternity hospitals linked to \\ the Rede Cegonha, Brazil - 2017
}

\author{
Silvana Granado Nogueira da Gama (https://orcid.org/0000-0002-9200-0387) ${ }^{1}$ \\ Elaine Fernandes Viellas (https://orcid.org/0000-0001-5259-8102) ${ }^{1}$ \\ Edymara Tatagiba Medina (https://orcid.org/0000-0001-7180-6704) ${ }^{2}$ \\ Antonia Angulo-Tuesta (http://orcid.org/0000-0002-3231-5918) ${ }^{3}$ \\ Carla Kristiane Rocha Teixeira da Silva (https://orcid.org/0000-0002-4167-9342) ${ }^{4}$ \\ Sara Delfino da Silva (https://orcid.org/0000-0002-9695-8146) ${ }^{5}$ \\ Yammê Ramos Portella Santos (https://orcid.org/0000-0002-1955-7779) ${ }^{1}$ \\ Ana Paula Esteves-Pereira (https://orcid.org/0000-0002-0236-2043) ${ }^{1}$
}

${ }^{1}$ Escola Nacional de Saúde Pública, Fiocruz. R. Leopoldo Bulhões 1480,

Manguinhos. 21041-210 Rio de Janeiro RJ Brasil. silvana.granado@gmail.com ${ }^{2}$ Faculdade de Enfermagem, Universidade do Estado do Rio de Janeiro. Rio de Janeiro RJ Brasil.

${ }^{3}$ Faculdade de Ceilândia, Universidade de Brasília. Brasília DF Brasil.

${ }^{4}$ Secretaria de Estado de

Saúde do Rio de Janeiro. Rio

de Janeiro RJ Brasil.

${ }^{5}$ Faculdade ICESP Brasília.

Brasília DF Brasil.

\begin{abstract}
This study aimed to assess whether nurses' presence in delivery care in maternity hospitals linked to the Rede Cegonha program promotes access to best obstetric practices during labor and delivery. We conducted an evaluative study in 2017 in all 606 SUS maternity hospitals that joined this strategic policy in all Brazilian states. We collected data from maternity hospital managers and puerperae. The analysis was performed at two levels: hospital with or without a nurse in delivery care; and professionals that attended vaginal delivery, whether doctors or nurses. We used best practices and interventions for vaginal deliveries and cesarean section rates as dependent variables. We included 5.016 subjects for analyses of vaginal deliveries and 9.692 to calculate cesarean section rates. Multiple regressions were adjusted for geographic region, maternity hospital size, and puerperae skin color and parity. Maternity hospitals with nurses in delivery care used more the partograph and less oxytocin, lithotomy, episiotomy, and cesarean section. Deliveries attended by nurses had more frequent use of the partograph and a lower likelihood of lithotomy and episiotomy. The inclusion of nurses in vaginal delivery care has successfully brought women closer to a more physiological and respectful delivery. Key words Nurse, Rede Cegonha, Best practices, Labor and birth
\end{abstract}

Resumo O objetivo foi avaliar se a presença da enfermeira na atenção ao parto em maternidades da Rede Cegonha promove o acesso às boas práticas de atenção obstétrica ao trabalho de parto e parto. Avaliação conduzida em 2017, nas 606 maternidades do SUS que aderiram a essa politica estratégica em todos os estados do Brasil. Utilizamos dados coletados com gestores das maternidades e puérperas. A análise ocorreu em dois níveis: hospitalar, com ou sem assistência ao parto por enfermeiras; e profissional, que assistiu ao parto vaginal, médico ou enfermeira. Como variáveis dependentes incluimos boas práticas e intervenções no parto vaginal e na taxa de cesariana. Foram incluidas na análise dos partos vaginais 5.016 puérperas e no cálculo da taxa de cesariana 9.692. Análises múltiplas foram ajustadas por região geográfica, localização, porte da maternidade, cor da pele e paridade da puérpera. Maternidades com enfermeira na assistência ao parto usam mais partograma; e menos ocitocina, litotomia, episiotomia e cesariana. Em partos assistidos por enfermeiras foi mais frequente o preenchimento do partograma e menor a chance de litotomia e episiotomia. A inserção da enfermeira na assistência ao parto vaginal tem se mostrado bem sucedida, trazendo às mulheres um parto mais fisiológico e respeitoso.

Palavras-chave Enfermeira, Rede Cegonha, Boas práticas obstétricas, Parto vaginal 


\section{Introdução}

Em meados do século XX ocorreu o processo de institucionalização do parto no Brasil, com atenção centrada no médico, retirando da mulher sua autonomia. De um processo natural o parto foi se tornando evento patológico, cada vez com mais intervenções ${ }^{1,2}$, incluindo a cesariana, que desde 2009 passou a ser a principal via de nascimento no país ${ }^{3}$.

Na década de 1980, o Ministério da Saúde, profissionais e organizações de mulheres iniciaram um movimento em busca de modelos de atenção ao parto que assegurassem a elas o direito ao planejamento reprodutivo, atenção de qualidade durante a gravidez, parto e puerpério4. Além de visar um nascimento seguro, com cuidado centrado na mulher, garantindo os princípios do Sistema Único de Saúde (SUS), que versam sobre a integralidade, universalidade, igualdade e organização dos diferentes níveis da atenção materno-infantil ${ }^{5}$.

Em 2011, o Ministério da Saúde instituiu a Rede Cegonha $(\mathrm{RC})^{6}$, política pública que busca implementar um modelo de atenção ao parto e ao nascimento com base nos princípios da humanização. Foi ampliada a oferta de formação e capacitação de enfermeiras obstetras e obstetrizes, implantados Centros de Parto Normal $(\mathrm{CPN})$, com ambientes propícios às boas práticas obstétricas, e promovida a inserção dessas profissionais na assistência ao parto vaginal sem distócia.

As estratégias adotadas pela RC são fortemente embasadas em evidências científicas que demonstram os benefícios da atenção ao parto por enfermeiras e obstetrizes, tanto para as mulheres quanto para os seus filhos ${ }^{7,8}$, levando ao aumento da satisfação, sem prejuízo aos indicadores perinatais?.

O objetivo deste estudo foi avaliar se a presença da enfermeira na atenção ao parto em maternidades da Rede Cegonha promove o acesso das gestantes às boas práticas de atenção obstétrica no trabalho de parto e parto.

\section{Métodos}

Este artigo utiliza dados da "Avaliação da Atenção ao Parto e Nascimento em Maternidades da Rede Cegonha", cuja coleta de dados se deu com base na técnica de Estimativa Rápida Participativa $\mathrm{ERP}^{10}$, recomendada pela Organização Pan-Americana de Saúde - OPS.
Foram incluídos na avaliação da RC todos os 606 hospitais públicos e mistos (privados conveniados ao SUS) que, em 2015, tinham plano de ação da RC e que representavam quase $50 \%$ dos partos ocorridos naquele ano no país, segundo sistema de informação sobre nascidos vivos - SINASC.

O trabalho de campo foi realizado entre dezembro de 2016 e outubro de 2017, incluindo a aplicação de cinco formulários eletrônicos específicos para cada fonte de dados, utilizando-se três diferentes métodos de coleta de dados: 1- entrevista pessoal com informantes-chaves: gestores; profissionais de saúde e puérperas, a fim de verificar a percepção dos mesmos quanto ao modelo de gestão e atenção ao parto e nascimento. 2- análise documental: para obter informações sobre a gestão dos serviços, processos de trabalho da equipe e organização do cuidado, verificar a disponibilidade de indicadores de processo e resultados da assistência ao parto e nascimento; 3- observação in loco, cuja finalidade foi avaliar processos de cuidado e as condições de infraestrutura, planta física, equipamentos, materiais, insumos e a contagem de leitos obstétricos e neonatais do hospital ${ }^{11}$.

$\mathrm{Na}$ avaliação da RC foram incluídas todas as puérperas com parto durante o período do estudo avaliativo, exceto aquelas com transtorno mental grave, que não compreendiam o idioma português; surdas; ou internadas na Unidade Intermediária ou Unidade de Terapia Intensiva no pós-parto. A amostra foi selecionada em dois estágios, sendo o primeiro composto pelos hospitais e o segundo pelas puérperas. Maiores detalhes sobre a metodologia do estudo estão disponíveis em Vilella et al. ${ }^{11}$

Para o presente estudo considerou-se apenas as puérperas que tiveram parto vaginal, a termo ( $\geq 37$ semanas gestacionais) e feto/RN com peso ao nascer $\geq 2.500 \mathrm{~g}$. Na análise das boas práticas e intervenções, para a exposição "profissional que assistiu ao parto", foram excluídas as mulheres que não souberam dizer o profissional que as atenderam no parto e as que referiram outros profissionais que não médico ou enfermeiro. Para a análise comparativa da frequência de cesarianas, foram inseridos todos os nascimentos nas maternidades estudadas.

Duas formas de análise foram empregadas. $\mathrm{Na}$ primeira, as mulheres foram comparadas considerando como variável de exposição o modelo de parto adotado na maternidade, com ou sem a atuação direta da enfermeira/obstetriz na assistência. Na segunda, a comparação se deu 
inserindo como variável de exposição o tipo de profissional que assistiu ao parto, se médico ou enfermeira/obstetriz.

Foram incluídas como covariáveis: macrorregião de residência da mãe; localização (capital/região metropolitana, demais municípios); tamanho da maternidade $(<500$ partos/ano, 500-1.499 partos/ano, 1.500-2.999 partos/ano, $\geq 3.000$ partos/ano); idade materna $(<20$ anos, 20-34 anos e $\geq 35$ anos); cor da pele (branca, preta, parda, amarela, indígena); situação conjugal (sem companheiro, com companheiro); nível de escolaridade ( $<$ fundamental, fundamental completo, $\geq$ ensino médio completo); paridade (primípara, 1 parto anterior, 2 partos anteriores, $\geq 3$ partos anteriores).

Como desfechos incluímos variáveis relacionadas às boas práticas durante o trabalho de parto (TP) (oferta de líquidos e alimentos, movimentação, métodos não farmacológicos para alívio da dor, uso de partograma); boas práticas no recém-nascido (aleitamento na sala de parto e contato pele a pele imediato); além das intervenções durante o TP (administração de soro por punção venosa, ocitocina, analgesia e amniotomia) e no parto (litotomia, manobra de Kristeller e episiotomia, além da cesariana). Para a análise da cesariana foram incluídos todos os nascimentos ocorridos naquelas maternidades. O tamanho da maternidade foi extraído do instrumento aplicado aos gestores e as demais variáveis do questionário aplicado às puérperas, com opção de resposta "sim e não".

Utilizamos o teste de qui-quadrado de Pearson $\left(\chi^{2}\right)$ para comparar as proporções das características das mulheres segundo o modelo de parto adotado no hospital (com ou sem enfermeira na assistência ao parto) e o profissional que as assistiu ao parto (médico ou enfermeira). Empregamos dois modelos de regressão logística para testar a associação entre o modelo de parto adotado no hospital e o profissional que prestou a assistência ao parto e as boas práticas e intervenções na assistência ao TP, parto e nascimento.

O nível de significância estabelecido foi de $5 \%$ e utilizamos estimativas de razão de chances (OR) considerando o efeito do desenho amostral. As OR foram ajustadas para macrorregião geográfica, localização, tamanho da maternidade, cor da pele e paridade da puérpera. Para as análises utilizamos um fator de calibração pelo inverso da probabilidade de inclusão de cada puérpera como garantia de que a distribuição das puérperas amostradas correspondesse à distribuição de partos ocorridos nas maternidades analisadas em
2017, e considerado o efeito de cluster em todas as análises. Os softwares empregados nas análises foram o SPSS 20.0 e Microsoft Excel versão 2007.

O estudo foi aprovado pelo Comitê de Ética em Pesquisa com Seres Humanos da Universidade Federal do Maranhão e da Escola Nacional de Saúde Pública Sérgio Arouca, em 14 de dezembro de 2016. A todas as mulheres foi lido o termo de consentimento livre e esclarecido (TCLE) e entregue uma cópia assinada àquelas que concordaram em participar da avaliação. Foram adotados os devidos cuidados para garantir o sigilo e a confidencialidade dos dados.

\section{Resultados}

Foram entrevistadas 10.665 puérperas no pós -parto imediato. Após aplicar os critérios de exclusão, permaneceram 571 maternidades. $\mathrm{Na}$ análise comparativa segundo o modelo de parto da maternidade foram incluídas 5.016 puérperas. Para a análise segundo profissional que assistiu ao parto foram ainda excluídas as mulheres que não souberam informar o profissional que as atendeu $(9,2 \%)$ e aquelas que relataram profissional diferente de médico ou enfermeira $(0,9 \%)$, restando 4.504 mulheres. Por fim, apenas para o cálculo da taxa de cesariana foram inseridos todos os 9.692 nascimentos ocorridos no conjunto de maternidades estudadas.

A distribuição dos partos atendidos por enfermeiras no Brasil difere tanto em relação às macrorregiões, quanto por localização e volume de partos da maternidade. No total das maternidades avaliadas, 30\% dos partos vaginais foram assistidos por enfermeiras, chegando a $46 \%$ no Norte do país. Por outro lado, as regiões Centro -Oeste e Sul detiveram as menores frequências, $19,6 \%$ e $18,5 \%$, respectivamente. A assistência ao parto por enfermeiras também foi mais realizada em municípios do interior, quando comparada às regiões metropolitanas e nas maternidades com $\geq 3.000$ partos por ano, em comparação às de menor porte (Gráfico 1).

Destaca-se o perfil semelhante das mulheres entrevistadas, seja comparando por tipo de profissional seja por tipo de modelo de parto da maternidade. Aproximadamente 23\% das mulheres eram adolescentes e $42 \%$ tinham ensino médio completo ou mais. Apenas a paridade as distinguiu em relação ao tipo de profissional que assistiu ao parto, sendo as primíparas mais atendidas pelos médicos. Quanto ao modelo de parto da maternidade, naquelas com assistência 


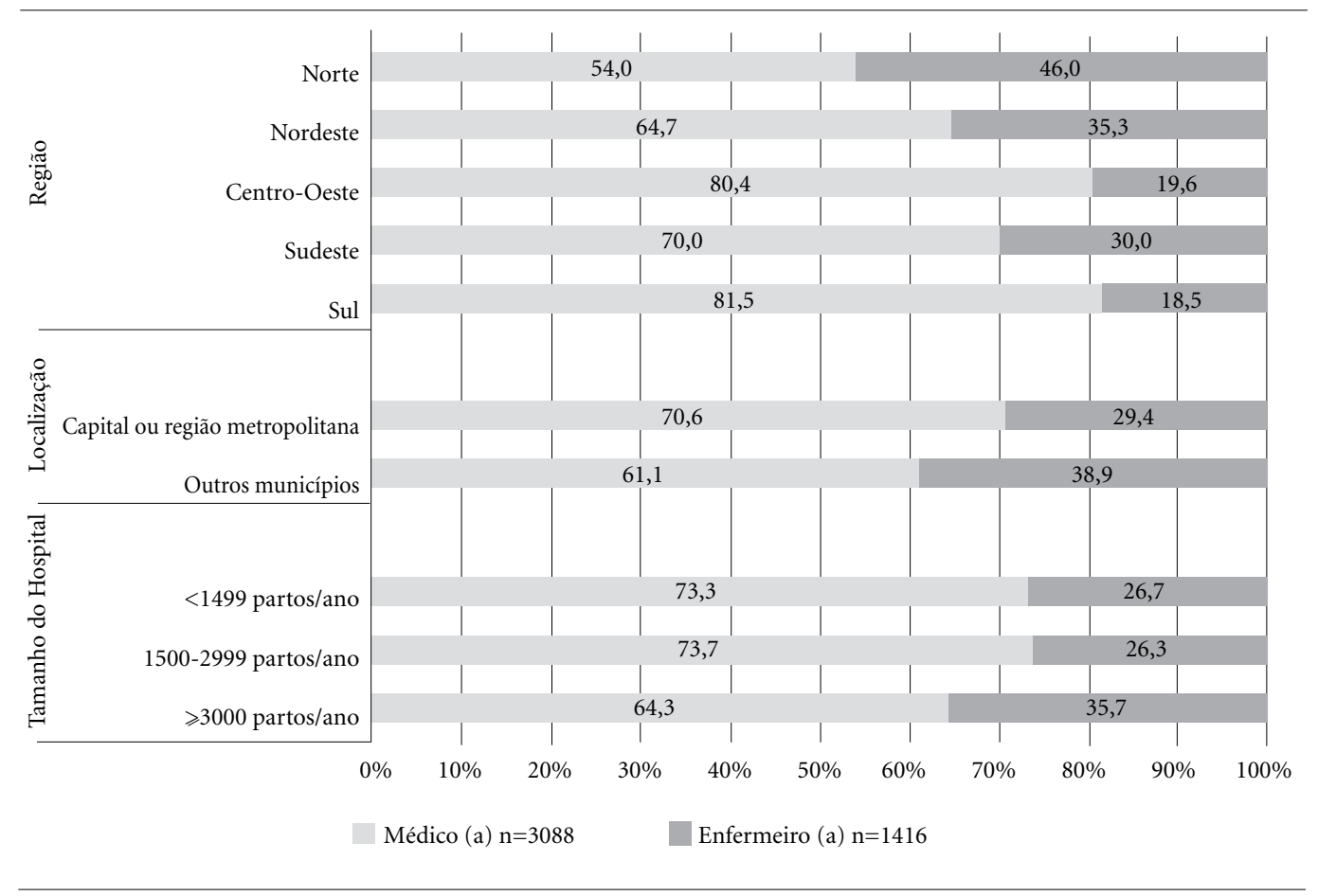

Gráfico 1. Proporção de partos vaginais assitidos por médico ou enfermeiro, segundo características hospitalares em maternidades da Rede Cegonha - Brasil, 2017.

por enfermeira, a cor da pele parda e viver com companheiro foram mais frequentemente relatadas (Tabela 1).

Nas 309 maternidades com assistência por enfermeiras, as boas práticas durante o $\mathrm{TP}$, parto e com o recém-nascido (RN) foram mais frequentes. Houve maior oferta de líquidos e alimentos, mais incentivo à movimentação da gestante, uso de pelo menos um método não farmacológico para alívio da dor, maior contato pele a pele do $\mathrm{RN}$ com a mãe e maior oferta do seio materno na sala de parto (Tabela 2).

Resultado semelhante foi encontrado na comparação por tipo de profissional que assistiu ao parto, com maior adesão às boas práticas e menor uso de intervenções obstétricas pelas enfermeiras do que pelos médicos. A amniotomia, que não foi diferenciada segundo o modelo de parto da maternidade, também foi significativamente menos praticada pelas enfermeiras (Tabela 2).

Algumas intervenções permanecem na rotina das maternidades estudadas. No entanto, foram menos realizadas em maternidades que contam com a participação direta da enfermeira na assistência ao parto. Nestas maternidades foi menos frequente a administração de soro por punção venosa, o uso de ocitocina e analgesia durante o TP; e, no parto, foi menor o uso da posição de litotomia, manobra de Kristeller e episiotomia. A proporção de cirurgia cesariana foi elevada nas maternidades avaliadas $(48,2 \%)$, mas foi menos praticada naquelas cujo modelo insere a enfermeira na assistência ao parto vaginal (Tabela 2).

$\mathrm{Na}$ análise múltipla, as maternidades com inserção da enfermagem obstétrica na assistência ao parto vaginal tiveram maior uso de partograma $(\mathrm{OR}=1,9$ IC 1,3-2,7) e menor de ocitocina $(\mathrm{OR}=0,7$ IC $0,5-0,9)$, litotomia $(\mathrm{OR}=0,6$ IC 0,4-0,9) e episiotomia (OR = 0,7 IC 0,5-0,9), em comparação àquelas sem inserção da enfermagem obstétrica, mesmo após ajustes pelas variáveis de confundimento. Ao analisar todos os nascimentos, as cesarianas também foram menos realizadas nestas maternidades $(\mathrm{OR}=0,8 \mathrm{IC} 0,7$ 0,9) (Tabela 3).

Ao considerar o tipo de profissional que assistiu ao parto (médico ou enfermeira), o resultado da análise múltipla indicou um maior preenchimento de partograma $(\mathrm{OR}=1,5 \mathrm{IC} 1,2-1,9)$ e menor uso de litotomia ( $\mathrm{OR}=0,7$ IC $0,5-0,8)$ e episiotomia $(\mathrm{OR}=0,4$ IC $0,3-0,6)$ pelas en- 
Tabela 1. Características maternas segundo modelo de parto da maternidade e tipo de profissional que assistiu ao parto vaginal em maternidades que aderiram à Rede Cegonha, Brasil, 2017.

\begin{tabular}{|c|c|c|c|c|c|c|c|c|}
\hline \multirow{2}{*}{ Variáveis } & \multicolumn{4}{|c|}{$\begin{array}{c}\text { Maternidades com assistência ao parto } \\
\text { vaginal por enfermeira }\end{array}$} & \multicolumn{4}{|c|}{$\begin{array}{c}\text { Tipo de profissional que assistiu ao parto } \\
\text { vaginal }\end{array}$} \\
\hline & $\begin{array}{c}\text { Não } \\
\mathrm{n}=1.135\end{array}$ & $\begin{array}{c}\text { Sim } \\
\mathbf{n}=\mathbf{3 . 8 8 1}\end{array}$ & $\begin{array}{c}\text { Total } \\
\mathbf{n}=5.016\end{array}$ & P-valor ${ }^{1}$ & $\begin{array}{c}\text { Médico } \\
\mathrm{n}=\mathbf{3 . 0 8 8}\end{array}$ & $\begin{array}{c}\text { Enfermeira } \\
\mathrm{n}=1.416\end{array}$ & $\begin{array}{c}\text { Total } \\
\mathbf{n}=\mathbf{4 . 5 0 4}\end{array}$ & P-valor ${ }^{2}$ \\
\hline & $(\%)$ & $(\%)$ & $(\%)$ & & $(\%)$ & $(\%)$ & $(\%)$ & \\
\hline \multicolumn{9}{|l|}{ Idade materna } \\
\hline$<20$ anos & 22,6 & 23,8 & 23,5 & 0,439 & 23,0 & 23,4 & 23,1 & 0,934 \\
\hline 20-34 anos & 68,3 & 68,4 & 68,4 & & 68,6 & 68,0 & 68,4 & \\
\hline$\geq 35$ anos & 9,1 & 7,9 & 8,1 & & 8,4 & 8,7 & 8,5 & \\
\hline \multicolumn{9}{|l|}{ Cor da pele } \\
\hline Branca & 33,2 & 25,1 & 26,9 & 0,003 & 28,2 & 24,3 & 27,0 & 0,063 \\
\hline Preta & 13,9 & 13,8 & 13,8 & & 13,8 & 13,0 & 13,6 & \\
\hline Parda & 52,9 & 61,1 & 59,2 & & 58,0 & 62,7 & 59,5 & \\
\hline \multicolumn{9}{|l|}{ Situação conjugal } \\
\hline Sem companheiro & 26,5 & 21,7 & 22,8 & 0,038 & 23,0 & 21,0 & 22,3 & 0,244 \\
\hline Com companheiro & 73,5 & 78,3 & 77,2 & & 77,0 & 79,0 & 77,7 & \\
\hline \multicolumn{9}{|l|}{ Escolaridade } \\
\hline$<$ Fundamental & 29,8 & 27,7 & 28,2 & 0,435 & 27,4 & 28,3 & 27,7 & 0,475 \\
\hline $\begin{array}{l}\text { Fundamental } \\
\text { completo }\end{array}$ & 30,1 & 29,5 & 29,6 & & 29,1 & 30,8 & 29,6 & \\
\hline $\begin{array}{l}\geq \text { Ensino médio } \\
\text { completo }\end{array}$ & 40,1 & 42,9 & 42,2 & & 43,5 & 41,0 & 42,7 & \\
\hline \multicolumn{9}{|l|}{ Paridade } \\
\hline Primípara & 49,1 & 47,1 & 47,6 & 0,692 & 49,1 & 42,1 & 46,9 & 0,001 \\
\hline Um parto anterior & 24,7 & 26,9 & 26,4 & & 26,2 & 27,3 & 26,5 & \\
\hline $\begin{array}{l}\text { Dois partos } \\
\text { anteriores }\end{array}$ & 13,3 & 13,1 & 13,2 & & 12,6 & 15,7 & 13,6 & \\
\hline $\begin{array}{l}\text { Três ou mais } \\
\text { partos anteriores }\end{array}$ & 12,9 & 12,9 & 12,9 & & 12,1 & 14,9 & 13,0 & \\
\hline
\end{tabular}

fermeiras, resultado semelhante à comparação segundo o modelo de parto da maternidade (Tabela 4$)$.

\section{Discussão}

A Organização Mundial da Saúde (OMS) designou 2020 como o "Ano da Enfermeira e da Enfermeira obstétrica/obstetriz", um reconhecimento destinado a chamar a atenção do mundo para o papel essencial que enfermeiras e parteiras têm na transformação da saúde de mulheres e bebês ${ }^{12}$. Os resultados deste estudo demonstram avanços significativos na atenção ao parto vaginal e melhoria das boas práticas nas maternidades públicas vinculadas à RC. Mais de um terço dos partos vaginais foram atendidos por enfermeiras, em contraste aos $16,2 \%$ encontrados nas ma- ternidades do SUS do estudo "Nascer no Brasil" 2011/12 ${ }^{13}$. Leal et al. ${ }^{14}$, na comparação da Rede Cegonha com hospitais públicos e mistos do estudo "Nascer no Brasil" 2011/12, observou redução nas intervenções durante o TP e parto e aumento das boas práticas, independentemente do profissional que prestou a assistência, sugerindo efeito positivo dessa política pública para alcançar os resultados esperados, que visam o parto/ nascimento respeitoso, seguro e digno, com base nas melhores evidências científicas.

Apesar dos avanços identificados, foi baixa a taxa de partos vaginais e, ainda insuficiente, a assistência destes partos por enfermeiras obstétricas. A legislação da enfermagem obstétrica no Brasil faculta, à mesma, a assistência ao parto de risco habitual desde a admissão à alta, e a identificação das distócias e tomada de decisão até a transferência do cuidado ao profissional médi- 
Tabela 2. Boas práticas e intervenções obstétricas segundo modelo de parto da maternidade e tipo de profissional que assistiu ao parto vaginal em maternidades que aderiram à Rede Cegonha, Brasil, 2017

\begin{tabular}{|c|c|c|c|c|c|c|c|c|}
\hline \multirow[t]{2}{*}{ Variáveis } & \multicolumn{4}{|c|}{$\begin{array}{c}\text { Maternidades com assistência ao } \\
\text { parto vaginal por enfermeiro }\end{array}$} & \multicolumn{4}{|c|}{$\begin{array}{c}\text { Tipo de profissional que assistiu o parto } \\
\text { vaginal }\end{array}$} \\
\hline & Não & Sim & Total & P-valor ${ }^{1}$ & Médico & Enfermeiro & Total & P-valor ${ }^{2}$ \\
\hline Número de maternidades & 262 & 309 & 571 & - & - & - & - & - \\
\hline Número de partos vaginais & 1244 & 3432 & 5.016 & - & 3088 & 1416 & 4.504 & - \\
\hline$\%$ dentre os partos vaginais & $\%$ & $\%$ & $\%$ & & $\%$ & $\%$ & $\%$ & \\
\hline \multicolumn{9}{|l|}{ Boas práticas durante o $\mathrm{TP}$} \\
\hline $\begin{array}{l}\text { Oferta de líquidos e } \\
\text { alimentos }\end{array}$ & 40,4 & 49,4 & 47,4 & 0,001 & 45,8 & 51,5 & 47,6 & 0,011 \\
\hline Movimentação & 70,7 & 77,7 & 76,1 & 0,002 & 74,8 & 79,8 & 76,3 & $<0,001$ \\
\hline $\begin{array}{l}\text { Métodos não farmacológicos } \\
\text { para alívio da dor }\end{array}$ & 64,5 & 70,5 & 69,2 & 0,055 & 67,9 & 72,6 & 69,4 & 0,032 \\
\hline Uso de partograma & 61,2 & 66,7 & 65,5 & 0,151 & 63,8 & 68,5 & 65,3 & 0,054 \\
\hline \multicolumn{9}{|l|}{$\begin{array}{l}\text { Boas práticas com o recém- } \\
\text { nascido }\end{array}$} \\
\hline $\begin{array}{l}\text { Aleitamento na sala de } \\
\text { parto }\end{array}$ & 34,1 & 44,1 & 41,9 & 0,005 & 39,1 & 47,0 & 41,6 & 0,002 \\
\hline $\begin{array}{l}\text { Contato pele a pele após } \\
\text { o parto }\end{array}$ & 59,5 & 71,1 & 68,5 & $<0,001$ & 66,9 & 73,0 & 68,8 & 0,003 \\
\hline \multicolumn{9}{|l|}{ Intervenções durante o TP } \\
\hline Soro por punção venosa & 67,4 & 55,9 & 58,5 & $<0,001$ & 61,7 & 51,1 & 58,4 & $<0,001$ \\
\hline Ocitocina & 47,2 & 36,2 & 38,7 & $<0,001$ & 41,9 & 32,3 & 38,9 & $<0,001$ \\
\hline Analgesia & 21,8 & 14,4 & 16,0 & 0,001 & 17,9 & 13,4 & 16,5 & 0,011 \\
\hline Amniotomia & 43,0 & 39,5 & 40,3 & 0,116 & 42,9 & 35,1 & 40,4 & $<0,001$ \\
\hline \multicolumn{9}{|l|}{ Intervenções durante o parto } \\
\hline $\begin{array}{l}\text { Litotomia (parto } \\
\text { horizontal) }\end{array}$ & 93,6 & 85,2 & 87,1 & $<0,001$ & 89,9 & 82,9 & 87,7 & $<0,001$ \\
\hline Manobra de Kristeler & 21,8 & 14,8 & 16,4 & $<0,001$ & 18,5 & 13,4 & 16,8 & $<0,001$ \\
\hline Episiotomia & 46,1 & 30,8 & 34,2 & $<0,001$ & 40,7 & 21,6 & 34,6 & $<0,001$ \\
\hline Cesariana $^{3}$ & 52,3 & 46,9 & 48,2 & 0,007 & - & - & - & - \\
\hline
\end{tabular}

1 P-valor de teste qui-quadrado na comparação entre hospital com ou sem enfermeiro na assistência ao parto. 2 P-valor de teste qui-quadrado na comparação parto realizado por enfermeiro e por médico. $3 \mathrm{~N}$ total $=9692$ puérperas.

$\mathrm{co}^{15}$. Esses achados revelam barreiras e dificuldades para a atuação da enfermagem obstétrica, que podem ser atribuídas ao investimento ainda insuficiente na formação dessa profissional, à baixa contratação pelos hospitais públicos, menor ainda pelos hospitais privados, e aos salários pouco atrativos. Soma-se ainda, a resistência dos obstetras para o trabalho colaborativo e as disputas expressas pelos conselhos profissionais de medicina federal e regionais ${ }^{16-19}$.

A atuação da enfermeira/obstetriz no Brasil, ainda guarda diferenças significativas quando comparada às experiências dos sistemas de saúde europeus, Reino Unido, Holanda e Alemanha e em outros países como Austrália, onde as enfermeiras/parteiras exercem protagonismo importante no acompanhamento da gestação e parto, cabendo aos médicos apenas a assistência aos partos cirúrgicos ou com complicações ${ }^{7,20-24}$.

$\mathrm{O}$ modelo de atenção proposto pela RC propõe mudanças no cuidado à saúde da mulher $\mathrm{e}$ da criança, trazendo como diretriz a garantia das boas práticas e segurança na atenção ao parto e nascimento. Uma das ações é o incentivo à maior participação da enfermeira na assistência prénatal, ao parto e nascimento, incluindo financiamento do Programa Nacional de Residência em Enfermagem Obstétrica (PRONAENF) e cursos de aprimoramento de enfermeiras obstétricas em todo o território nacional. Essas diretrizes da RC facultaram a ampliação da assistência à mulher pela enfermagem obstétrica/obstetriz, contribuindo para a maior oferta de boas práticas no cuidado ao parto e diminuição das intervenções ${ }^{6,25}$. 
Tabela 3. Associação entre as práticas obstétricas e as maternidades com participação da enfermeira na assistência ao parto em maternidades que aderiram à Rede Cegonha, Brasil, 2017.

\begin{tabular}{|c|c|c|c|c|c|c|c|c|}
\hline \multirow{2}{*}{$\begin{array}{c}\text { Variáveis } \\
\text { Boas práticas durante o trabalho de parto }\end{array}$} & \multirow[t]{2}{*}{$\begin{array}{c}\text { OR } \\
\text { bruta }\end{array}$} & \multicolumn{3}{|c|}{$\begin{array}{c}\text { IC } 95 \% \\
\text { (OR bruta) }\end{array}$} & \multirow[t]{2}{*}{$\begin{array}{c}\text { OR } \\
\text { ajustada* }^{*}\end{array}$} & \multicolumn{3}{|c|}{$\begin{array}{c}\text { IC } 95 \% \\
\text { (OR ajustada) }\end{array}$} \\
\hline & & & & & & & & \\
\hline Oferta de líquidos e alimentos & 1,4 & 1,2 & - & 1,8 & 1,2 & 1,0 & - & 1,5 \\
\hline Movimentação & 1,4 & 1,1 & - & 1,8 & 1,0 & 0,7 & - & 1,3 \\
\hline $\begin{array}{l}\text { Uso de procedimentos não farmacológicos para alívio } \\
\text { da dor }\end{array}$ & 1,3 & 1,0 & - & 1,7 & 1,3 & 0,9 & - & 1,7 \\
\hline Uso de partograma & 1,3 & 1,0 & - & 1,8 & 1,9 & 1,3 & - & 2,7 \\
\hline \multicolumn{9}{|l|}{ Boas práticas com o recém-nascido } \\
\hline Aleitamento na sala de parto & 1,5 & 1,1 & - & 2,0 & 1,1 & 0,8 & - & 1,5 \\
\hline Contato pele a pele após o parto & 1,7 & 1,3 & - & 2,2 & 1,3 & 1,0 & - & 1,7 \\
\hline \multicolumn{9}{|l|}{ Intervenções durante o trabalho de parto } \\
\hline Soro por punção venosa & 0,6 & 0,5 & - & 0,8 & 0,9 & 0,7 & & 1,2 \\
\hline Ocitocina & 0,6 & 0,5 & - & 0,8 & 0,7 & 0,5 & & 0,9 \\
\hline Analgesia & 0,6 & 0,5 & - & 0,8 & 0,7 & 0,5 & - & 1,0 \\
\hline Amniotomia & 0,9 & 0,7 & - & 1,0 & 1,0 & 0,8 & - & 1,2 \\
\hline \multicolumn{9}{|l|}{ Intervenções durante o parto } \\
\hline Litotomia (parto horizontal) & 0,4 & 0,3 & - & 0,6 & 0,6 & 0,4 & & 0,9 \\
\hline Manobra de Kristeller & 0,6 & 0,5 & - & 0,8 & 0,9 & 0,7 & - & 1,1 \\
\hline Episiotomia & 0,5 & 0,4 & - & 0,6 & 0,7 & 0,5 & & 0,9 \\
\hline Cesariana $^{* *}$ & 1,3 & 1,1 & - & 1,5 & 0,8 & 0,7 & 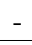 & 0,9 \\
\hline
\end{tabular}

${ }^{\star}$ Controlado por região geográfica, localização e tamanho do hospital. ${ }^{* \star}$ Modelo somente com cesariana.

Tabela 4. Associação entre as práticas obstétricas e a assistência ao parto realizada por enfermeira em maternidades que aderiram à Rede Cegonha, Brasil, 2017.

\begin{tabular}{|c|c|c|c|c|c|c|c|c|}
\hline \multirow{2}{*}{ Boas práticas durante o trabalho de parto } & \multirow[t]{2}{*}{$\begin{array}{c}\text { OR } \\
\text { bruta }\end{array}$} & \multicolumn{3}{|c|}{$\begin{array}{c}\text { IC } 95 \% \\
\text { (OR bruta) }\end{array}$} & \multirow[t]{2}{*}{$\begin{array}{c}\text { OR } \\
\text { ajustada* }^{*}\end{array}$} & \multicolumn{3}{|c|}{$\begin{array}{c}\text { IC } 95 \% \\
\text { (OR ajustada) }\end{array}$} \\
\hline & & & & & & & & \\
\hline Oferta de líquidos e alimentos & 1,3 & 1,1 & - & 1,5 & 1,1 & 0,9 & - & 1,3 \\
\hline Movimentação & 1,3 & 1,1 & - & 1,6 & 1,0 & 0,8 & - & 1,2 \\
\hline $\begin{array}{l}\text { Uso de procedimentos não-farmacológicos para } \\
\text { alívio da dor }\end{array}$ & 1,3 & 1,0 & - & 1,7 & 1,2 & 1,0 & - & 1,5 \\
\hline Uso de partograma & 1,3 & 1,0 & - & 1,5 & 1,5 & 1,2 & - & 1,9 \\
\hline \multicolumn{9}{|l|}{ Boas práticas com o recém-nascido } \\
\hline Aleitamento na sala de parto & 1,4 & 1,1 & - & 1,7 & 1,2 & 1,0 & - & 1,5 \\
\hline Contato pele a pele após o parto & 1,3 & 1,1 & - & 1,6 & 1,1 & 0,9 & - & 1,4 \\
\hline \multicolumn{9}{|l|}{ Intervenções durante o trabalho de parto } \\
\hline Soro por punção venosa & 0,6 & 0,5 & - & 0,8 & 0,9 & 0,7 & - & 1,1 \\
\hline Ocitocina & 0,7 & 0,5 & - & 0,8 & 0,8 & 0,6 & - & 1,0 \\
\hline Analgesia & 0,7 & 0,6 & - & 0,9 & 1,0 & 0,7 & - & 1,3 \\
\hline Amniotomia & 0,7 & 0,6 & - & 0,9 & 0,8 & 0,7 & - & 1,0 \\
\hline \multicolumn{9}{|l|}{ Intervenções durante o parto } \\
\hline Litotomia (parto horizontal) & 0,5 & 0,4 & - & 0,7 & 0,7 & 0,5 & - & 0,8 \\
\hline Manobra de Kristeller & 0,7 & 0,6 & - & 0,8 & 0,9 & 0,7 & - & 1,2 \\
\hline Episiotomia & 0,4 & 0,3 & - & 0,5 & 0,4 & 0,3 & - & 0,6 \\
\hline
\end{tabular}


A proporção da enfermagem na condução do parto vaginal se deu de forma heterogênea segundo as características das maternidades. Apesar de possuir o menor volume de partos no país, na região Norte quase metade dos partos vaginais foram assistidos por enfermeiras; o que também foi observado nos municípios do interior, com quase $40 \%$. Esses dados apontam que ao se distanciar dos grandes centros urbanos e das capitais, a chance de a mulher ser atendida por uma enfermeira no momento do parto aumenta. Uma hipótese provável para tal achado seria a menor oferta de médicos nessas áreas.

Estudo realizado sobre a distribuição de médicos no país apontou a região Norte com a menor razão médico/habitantes, 1,12 médico a cada 100.000 habitantes, enquanto no Sudeste essa razão foi de 2,81. Pressupõe-se uma série de elementos, sendo o mais provável a atração exercida pelo fator econômico ${ }^{26}$. Em contraste, na região Sudeste, mesmo com ampla oferta de médicos, é crescente a participação de enfermeiras no parto, atrelada à mudança no modelo obstétrico, conforme recomendado pelo $\mathrm{MS}^{6}$ e pela $\mathrm{OMS}^{12}$.

Identificamos que nos hospitais de maior porte foi superior o percentual de partos atendidos por enfermeira. Priorizar hospitais pela maior concentração de partos vem sendo uma prática das políticas públicas instituídas na última década pelo Ministério da Saúde, visando alcançar o maior número de profissionais e mulheres, ao dar apoio e incentivos para a qualificação da atenção ao parto e nascimento, introduzindo modelo de parto colaborativo, incluindo enfermeiras obstétricas, conforme diretrizes nacionais ${ }^{6}$. Ainda podemos considerar a relevância destes grandes hospitais como centros irradiadores de políticas públicas, por serem polos regionais e de referência do SUS.

Em estudo prévio da avaliação da RC não se observou redução na taxa de cesariana em mulheres com pagamento público do parto, que se manteve por volta de $43 \%$ no período de 2011 a $2017^{14}$. Ainda que esse não fosse um objetivo direto da RC, trata-se de uma intervenção, muitas vezes, desnecessária, com mais chance de complicações à mulher e ao feto ${ }^{27,28}$. Em contrapartida, encontramos menor chance de cesariana para as mulheres que tiveram seus partos em maternidades que adotam modelo de parto com enfermeiras no atendimento ao parto vaginal. Betrán et al. ${ }^{29}$ demonstram que o uso excessivo de cesarianas, além de não mostrar benefícios às mulheres podem ampliar o risco de danos. Apontam ainda que os cuidados liderados por parteiras foram associados a proporções mais elevadas de partos fisiológicos, resultados mais seguros e de menor custo.

Estudos sobre a contribuição da enfermagem para a redução das taxas de cesariana demonstram resultados que variam da manutenção até a redução das taxas ${ }^{30-32}$. Segundo Johantgen et al.$^{30}$, essas diferenças com frequência atribuem-se ao viés de seleção, assumindo que mulheres que "necessitam" de cesariana têm maior probabilidade de serem atendidas por obstetras, enquanto as que decidem pelo parto com menos intervenções têm maior probabilidade de serem atendidas por enfermeiras ou obstetrizes. Em contraste, Edmonds et al. ${ }^{32}$ demonstraram variações de $8,3 \%$ até $48,0 \%$ nas taxas de cesáreas entre $3.031 \mathrm{mu}$ lheres cuidadas por enfermeiras e médicos no TP e parto e essas variações não estiveram associadas às condições maternas estudadas (nulíparas, a termo, feto único e apresentação cefálica), mas aos cuidados oferecidos às mulheres por enfermeiras. Os autores consideraram este achado como um potencial fator de enfermagem, que pode influenciar a probabilidade de cesárea.

Além disso, em estudo conduzido por Hildingsson et al.$^{33}$, foi observado que a presença de uma enfermeira habilitada diminui a ansiedade e o medo durante o processo de parto e nascimento. Acreditamos que estudos nesta temática devam ser estimulados, proporcionando melhores evidências sobre os efeitos deste cuidado. No presente estudo expomos benefícios da presença da enfermeira na assistência ao parto, evidenciando as vantagens de um modelo de parto incluindo essas profissionais.

Chama a atenção o fato de $9,2 \%$ das puérperas não saberem qual profissional prestou assistência ao seu parto vaginal. Apresentar-se à parturiente, informando nome e função, é um dos itens destacados na diretriz "acolhimento em obstetrícia” da RC. Dentre as entrevistadas, apenas $58,9 \%$ responderam que todos ou a maioria dos profissionais se apresentaram a elas ao longo da internação, fato que classificou o conjunto de maternidades como "parcialmente adequadas", segundo a matriz de avaliação da $\mathrm{RC}^{34}$.

Outras boas práticas na assistência ao TP e parto também se revelaram importantes. A utilização do partograma, no nível individual e da maternidade, foi maior onde havia atuação da enfermeira, o que se revela como indicador da prestação de cuidados e do registro das suas práticas, porque proporciona mais segurança à parturiente, promove a troca de informações entre profissionais e registra o histórico da atenção. 
Nesse sentido, configura-se como dispositivo tecnológico para o fortalecimento dos sistemas de informação nas maternidades e em nível nacional, que possibilitam o monitoramento e a avaliação da RC e dos progressos da atenção à saúde sexual e reprodutiva das mulheres no país, quanto à Meta 3.7 da Agenda do Desenvolvimento Sustentável $\mathrm{l}^{35}$.

A RC é composta por diretrizes que incentivam, além da adoção de boas práticas no TP e parto, como uso de métodos não farmacológicos para alívio da dor, o aleitamento materno e o fortalecimento do vínculo mãe-bebê. Ainda que tenha perdido significância estatística após o ajuste pelas variáveis de confundimento, destacamos uma provável maior prática de aleitamento materno na sala de parto e de contato pele a pele em hospitais que possuem enfermeiras atuantes no processo de nascimento, confirmando os esforços dessas profissionais no cumprimento das diretrizes da RC.

No que diz respeito às práticas no TP e parto que deveriam ser evitadas, o uso da ocitocina como rotina, a posição de litotomia e a episiotomia foram menos realizadas na presença de enfermeiras na assistência ao parto. Este resultado é consistente com outros estudos que demonstram menor uso dessas intervenções pelas enfermei$\operatorname{ras}^{22-24,30}$. Entretanto, a permanência dessas práticas tem implicações desfavoráveis, e reforça a necessidade de aperfeiçoamento da gestão hospitalar, para garantir a segurança e qualidade da atenção materna e neonatal nas maternidades, assegurando acesso equânime para as mulheres e melhorando o impacto econômico para o sistema de saúde ${ }^{36-39}$.
É urgente o fortalecimento e o investimento no sistema de saúde e nas políticas orientadas às mulheres que ofereçam suporte à saúde e direitos reprodutivos e ampliação da qualidade da atenção materna e neonatal. Inclui-se, ainda, a valorização dos trabalhadores de saúde, o aprimoramento da estrutura hospitalar e dos sistemas de informação e uma relação mais inclusiva, dando voz às mulheres ${ }^{40}$.

\section{Conclusões}

Os resultados aqui apresentados confirmam a assistência da enfermagem como menos intervencionista no parto, além de expor também o avanço das boas práticas exercidas pelos profissionais médicos em relação a estudo semelhante realizado no Brasil em 2011/12 ${ }^{13}$. Sugere uma influência positiva do trabalho colaborativo entre enfermeiras e obstetras na obtenção de melhores resultados e na humanização na atenção ao TP e parto de mulheres de risco habitual e seus neonatos. Estratégias globais assinalam iniciativas de aprimoramento da prática para melhorar o desempenho de enfermeiras e obstetrizes a partir de treinamento, educação e formação a fim de influenciar no modo de nascimento. No entanto, análises destas iniciativas apontam a necessidade de enfrentar barreiras profissionais relacionadas às iniquidades de gênero; questões econômicas, sociais e culturais de reconhecimento da atuação e profissionalização das enfermeiras e obstetrizes que lhe conferem um menor status sociocultural ${ }^{7,8,39,41,42}$.

\section{Colaboradores}

SGN Gama contribuiu com a ideia e todo o processo de elaboração do artigo. Levantou bibliografia, foi a principal redatora, especialmente da discussão e fez a última revisão. EF Viellas e CKRT Silva participaram da concepção e metodologia do artigo, análise e interpretação dos dados, e redação do texto. ET Medina, A AnguloTuesta e SD Silva participaram da análise e interpretação dos dados, e redação do texto. YRP Santos criou as variáveis, rodou todas as tabelas e gerou o gráfico, contribuiu na metodologia do artigo. AP Esteves-Pereira realizou a revisão crítica de todo o manuscrito, incluindo o método de análise dos dados. Todas as autoras avaliaram e aprovaram a versão final encaminhada. 


\section{Referências}

1. Progianti JM, Barreira IA. A obstetrícia, do saber feminino à medicalização: da época medieval ao século XX. Rev enferm UERJ 2001; 9(1):91-97.

2. Nagahama EEI, Santiago SM. A institucionalização médica do parto no Brasil. Cien Saude Colet 2005; 10(3):651-657.

3. Brasil. Ministério da Saúde (MS). Datasus: informações de saúde: estatísticas vitais: nascidos vivos [página na Internet]. [acessado 2020 mai 9]. Disponível em: http://tabnet.datasus.gov.br/cgi/tabcgi.exe?sinasc/ cnv/nvuf.def

4. Rattner D. Humanização na atenção a nascimentos e partos: ponderações sobre políticas públicas. Interface (Botucatu) 2009; 13(Supl. 1):759-768.

5. Edmonds JK, Ivanof J, Kafulafula U. Midwife Led Units: Transforming maternity care globally. Annals of Global Health 2020; 86(1):44.

6. Brasil. Ministério da Saúde (MS). Portaria no 1.459, de 24 de junho de 2011. Institui, no âmbito do SUS, a Rede Cegonha. Diário Oficial da União 2011; 24 jun.

7. World Health Organization (WHO). Nursing and midwifery progress report 2008-2012. Geneve: WHO; 2013.

8. United Nations Population Fund (UNFPA). The state of the World's Midwifery 2014. A Universal Pathway. A woman's right to health. New York: UNFPA; 2014.

9. Sandall J, Soltani H, Gates S, Shennan A, Devane D. Midwifery-led continuity models versus other models of care for childbearing women. Cochrane Database Syst Rev 2016; (4):CD004667.

10. Piovesan MF, Padrão MVV, Dumont MU, Gondim GM, Flores O, Pedrosa JI, Lima LFM. Vigilância Sanitária: uma proposta de análise dos contextos locais. Rev. bras. Epidemiol 2005; 8(1):83-95.

11. Vilela MEA, Leal MC, Thomaz EBAF, Gomes MAM, Bittencourt S, Gama SGN, Silva L, Lamy Z. Avaliação da atenção ao parto e nascimento nas maternidades da Rede Cegonha: os caminhos metodológicos. Cien Saude Coletiva 2020; 26(3):789-800.

12. World Health Organization (WHO). WHO Campaigns. Year of the nurse and midwife 2020. Geneve: WHO; 2020.

13. Gama SGN, Viellas EF, Torres JA, Bastos MH, Brüggemann, Theme Filha MM, Schilithz AOC, Leal MC. Labor and birth care by nurse with midwifery skills in Brazil. Reproductive Health 2016; 3(Supl. 3):123.

14. Leal MC, Bittencourt AS, Esteves-Pereira AP, Ayres BVS, Silva LBRAA, Thomaz EBAF, Lamy ZC, Nakamura-Pereira M, Torres JA, Gama SGN, Domingues RMSM, Vilela MEA. Avanços na assistência ao parto no Brasil: resultados preliminares de dois estudos avaliativos. Cad. Saude Publica 2019; 35(7):e00223018.

15. Conselho Federal de Enfermagem (COFEN). Resolução no 516, de 24 de junho de 2016. Normatiza a atuação e a responsabilidade do Enfermeiro, Enfermeiro Obstetra e Obstetriz na assistência às gestantes, parturientes, puérperas e recém-nascidos nos Serviços de Obstetrícia, Centros de Parto Normal e/ou Casas de Parto e outros locais onde ocorra essa assistência; estabelece critérios para registro de títulos de Enfermeiro Obstetra e Obstetriz no âmbito do Sistema Cofen/Conselhos Regionais de Enfermagem, e dá outras providências. Diário Oficial da União 2016; 27 jun.
16. Angulo-Tuesta A, Giffin K, Gama AS, d'Orsi E, Barbosa GP. Saberes e práticas de enfermeiros e obstetras: cooperação e conflito na assistência ao parto. Cad Saude Publica 2003; 19(5):1425-1436.

17. Conselho Federal de Medicina (CFM). Parecer no 251, de 28 de maio de 1998. Aprova a realização de parto sem distocia por enfermeiro obstetra ou obstetriz. [página na Internet]. [acessado 2020 Abr 22]. Disponível em: https://sistemas.cfm.org.br/normas/visualizar/despachos/BR/1998/251

18. Conselho Federal de Medicina (CFM). Despacho COJUR/CFM n 814, de 7 de dezembro de 2018. Extrapolação dos limites legais na regulamentação da atividade de enfermeiras obstétricas nas casas de parto. [página na Internet]. [acessado 2020 Abr 22]. Disponível em: https://sistemas.cfm.org.br/normas/ visualizar/despachos/BR/2018/814

19. Conselho Regional de Medicina do Estado de Santa Catarina (CRM-SC). Parecer no 2486 / 2016. Enfermeira obstetriz e doulas são profissões regidas por legislação próprias e devem se ater a elas. Ao médico compete respeitar a decisão do paciente em relação ao parto, desde que sejam sem complicações. [página da Internet]. [acessado $2020 \mathrm{Fev} 13$ ]. Disponível em: https://sistemas.cfm.org.br/normas/visualizar/pareceres/SC/2016/2486

20. National Maternity Review. Better births: improving outcomes of maternity services in England. NHS England; 2016. [acessado 2020 mai 23]. Disponível em: https://www.england.nhs.uk/wp-content/uploads/2016/02/national-maternity-review-report.pdf

21. Homer CSE, Friberg IK, Dias MAB, Hoope-Bender PT, Sandall J, Speciale AM, Bartlett LA. The projected effect of scaling up midwifery. Lancet 2014; 384(9948):1146-1157.

22. Renfrew MJ, McFadden A, Bastos MH, Campbell J, Channon AA, Cheung NF, Silva DRAD, Downe S, Kennedy HP, Malata A, McCormick F, Wick L, Declercq E. Midwifery and quality care: findings from a new evidence-informed framework for maternal and newborn care. Lancet 2014; 384(9948):1129-1145.

23. Sandall J, Soltani H, Gates S, Shennan A, Devane D. Midwife-led continuity models versus other models of care for childbearing women. Cochrane Database of Systematic Reviews 2013; (8):CD004667.

24. Berg M, Ólafsdóttir OA, Lundgren I. A midwifery model of woman-centred childbirth care - In Swedish and Icelandic settings. Sex Reprod Healthc 2012; 3(2):79-87.

25. Santos Filho SB, Souza KV. Metodologia para articular processos de formação-intervenção-avaliação na educação profissional em enfermagem. Cien Saude Colet 2020; 25(1):79-88.

26. Póvoa L, Andrade MV. Distribuição geográfica dos médicos no Brasil: uma análise a partir de um modelo de escolha locacional. Cad Saude Publica 2006; 22(8):1555-1564.

27. Liu S, Liston RM, Joseph KS, Heaman M, Sauve R, Kramer MS. Maternal mortality and severe morbidity associated with low-risk planned cesarean delivery versus planned vaginal delivery at term. CMAJ 2007; 176(4):455-460. 
28. Kallianidis AF, Schutte JM, Van Roosmalen J, Van Den Akker T. Maternal mortality and severe morbidity audit committee of the Netherlands Society of Obstetrics and Gynecology. Maternal mortality after cesarean section in The Netherlands. Eur J Obstet Gynecol Reprod Biol 2018; 229:148-152.

29. Betrán AP, Temmerman M, Kingdon C, Mohiddin A, Opiyo N, Torloni MR, Zhang J, Musana O, Wanyonyi SZ, Gülmezoglu AM, Downe S. Interventions to reduce unnecessary caesarean sections in healthy women and babies. Lancet 2018; 392(10155):13581368.

30. Johantgen M, Fountain L, Zangaro G, Newhouse R, Stanik-Hutt J, White K. Comparison of labor and delivery care provided by certified nurse-midwives and physicians: A Systematic Review, 1990 to 2008. Women5s Health Issues 2012; 22(1):e73-e81.

31. Rosenstein MG, Nijagal M, Nakagawa S, Gregorich SE, Kuppermann M. The Association of Expanded Access to a Collaborative Midwifery and Laborist Model With Cesarean Delivery Rates. Obstet Gynecol 2015; 126(4):716-723.

32. Edmonds JK, O’Hara M, Clarke SP, Shah NT. Variation in Cesarean Birth Rates by Labor and Delivery Nurses. JOGNN 2017; 46:486-493.

33. Hildingssona I, Rubertssona C, Karlströmb A, Hainesa $\mathrm{H}$. Known midwife can make a difference for women with fear of childbirthbirth outcome and women's experiences of intrapartum care. Sex Reprod Healthc 2019; 21:33-38.

34. Bittencourt SDA, Vilela MEA, Marques MC, Santos A, Silva CK, Domingues RMSM, Reis A, Santos G. Atenção ao Parto e Nascimento em Maternidades da Rede Cegonha: avaliação do grau de implantação das ações. Cien Saude Coletiva 2020; 26(3):801-822.

35. Starrs AM, Ezeh AC, Barker G, Basu A, Bertrand JT, Blum R, Coll-Seck AM, Grover A, Laski L, Roa M, Sathar ZA, Say L, Serour GI, Singh S, Stenberg K, Temmerman M, Biddlecom A, Popinchalk A, Summers C, Ashford LS. Accelerate progress sexual and reproductive health and rights for all: report of the Guttmacher-Lancet Commission. Lancet 2018; 391(10140):2642-2692.

36. Kozhimannil BK, Law MR, Virnig BA. Cesarean delivery rates vary tenfold among US hospitals. Reducing variation may address quality and cost issues. Health Affairs 2013; 32(3):527-535.

37. Snowden JM, Darney BG, Cheng YW, McConnell KJ, Caughey AB. Systems factors in obstetric care: the role of daily obstetric volume. Obstet Gynecol 2013; 122(4):851-857.

38. Kenny C, Devane D, Normand C, Clarke M, Howard A, Begley C. A cost-comparison of midwife-led compared with consultant-led maternity care in Ireland (the MidU study). Midwifery 2015; 31(11):1032-1038.

39. Sharma G, Mathai M, Dickson KE, Weeks A, Hofmeyr GJ, Lavender T, Day LT, Mathews JE, Fawcus S, Simen -Kapeu A, de Bernis L. Quality care during labour and birth: a multi-country analysis of health system bottlenecks and potential solutions. BMC Pregnancy Childbirth 2015; 15(Supl. 2):S2.
40. Leal NP, Versiani MH, Leal MC, Portella Y. Práticas sociais do parto e do nascer no Brasil: a fala das puérperas. Cien Saude Coletiva 2020; 26(3):941-950.

41. Filby A, McConville F, Portela A. What Prevents Quality Midwifery Care? A systematic mapping of barriers in low and middle income countries from the provider perspective. PLoS ONE 2016; 11(5):e0153391.

42. ten Hoope-Bender P, de Bernis L, Campbell J, Downe S, Fauveau V, Fogstad H, Homer CS, Kennedy HP, Matthews Z, McFadden A, Renfrew MJ, Van Lerberghe W. Improvement of maternal and newborn health through midwifery. Lancet 2014; 384(9949):12261235 .

Artigo apresentado em 06/06/2020

Aprovado em 03/08/2020

Versão final apresentada em 05/08/2020

Editores-chefes: Romeu Gomes, Antônio Augusto Moura da Silva 
